\title{
Software Approach to Access UWB Interference on GPS Receivers
}

\author{
Y.T. Morton, M.P. French, Q. Zhou \\ Miami University \\ $\&$ \\ J.B.Y. Tsui, D.M. Lin, M.M. Miller, D. Janning \\ Air Force Research Laboratory
}

\section{ABSTRACT}

Ever since the FCC approved the use of UWB devices in commercial and federal bands, various agencies whose operations and/or products rely on the integrity of signals within certain "restricted" radio frequency bands have voiced concerns over the potential impact of the UWB interference. GPS signals are among these "restricted" bands. Several groups in the GPS community have conducted experimental studies concerning the impact of UWB interference on the performance of various grades of commercial and aviation GPS receivers.

In this paper, we present a software approach to simulate and evaluate UWB interference on GPS receivers. The software approach provides greater flexibility in the design of testing scenarios, such as the inclusion of a large number of aggregated UWB devices, the generation of new UWB signals and modulation schemes, and the possibility of extending the study to new GPS signals. The paper will discuss a general framework for developing algorithms to evaluate UWB and GPS interference under a wide variety of hardware and software conditions. This framework consists of three classes of components: Input, Processing, and Analysis. The input components are responsible for the generation of UWB signal waveforms and modulation schemes, and GPS signals. The processing components include a simulated model of GPS RF front end and software implementation of GPS processing blocks, such as acquisition, tracking, and post-processing. The Analysis components focus on the study of specific receiver processing component outputs. Both real and simulated

Authors' Current Addresses:

Y.T. Morton, M.P. French and Q. Zhou, School of Engineering and Applied Science, Miam University, Oxford, OH 45056, USA; and J.B.Y. Tsui, D.M. Lin, M.M. Miller and D. Janning, Sensors Directorate, Air Force Research Laboratory, Wright Patterson Air Force Base, OH 45433, USA.

Based on a presentation at PLANS 2004.

0885/8985/05/\$17.00 @ 2005 IEEE
UWB signals can be used in the study. The real UWB signals are primarily used to validate the simulation procedure, whereas the simulated UWB signals are used to allow the immediate incorporation of new UWB waveforms and modulations in the evaluations.

This paper will present details of the software components developed and the preliminary results achieved.

\section{INTRODUCTION}

In February 2002, the Federal Communication Commission (FCC) approved a Report \& Order $(\mathrm{R} \& \mathrm{O})$ that permitted the commercial use of the uitra-wideband (UWB) technology [1]. Since then, UWB has attracted considerable attention from communications and navigations communities because of several unique advantages as well as potential concerns that are associated with UWB signals. With the potential to offer data transmission rates of 100-500 Mbps at distances of 2-10 meters using average radiated power of a few hundred microwatts, and the possibility of miniaturized mass production at low cost, UWB technology is currently seen by many to be the backbone of future short-range wireless communication systems $[2,3]$. In addition, UWB signals have been utilized in imaging radar techniques because of their wall penetration capability, and are being studied for indoor location and navigation purposes because of their performance in multipath environments. With all of these potential applications and advantages, a mass market of many varieties of UWB devices may not be far in the future.

The question remains of the impact of a large number of active UWB devices on existing spectral users, especially for users of the Global Positioning Systems (GPS) whose signal power is far below the noise floor. Several groups have conducted studies of UWB interference and its impact on GPS receiver performance $[4,5,6,7]$. These studies used various grades of commercial GPS receivers and adopted several approaches in their testing. All of the testing has concluded that UWB signals degrade GPS receiver performance. There are, 


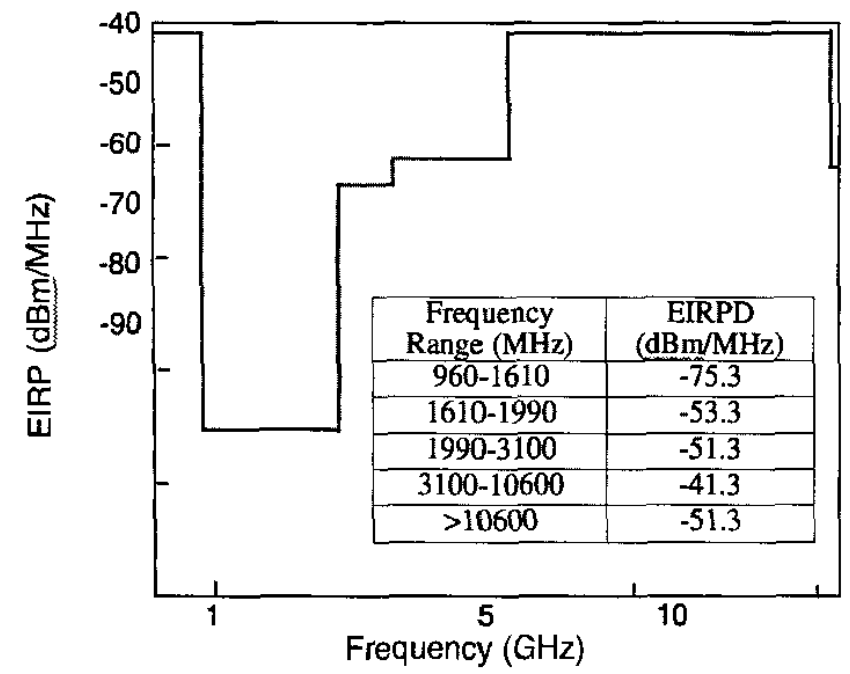

Fig. 1. Maximum Allowable UWB EIRPD Set By FCC in 2002

however, some unanswered questions and certain drawbacks associated with these testings. First, a wide variety of hardware and receiver architecture were used in the testing. The differences in the hardware made it difficult to determine the dominant factors that contribute to the degradation of receiver performance under UWB interference. Second, most tests were concerned with the errors in receiver pseudorange output or the amount of UWB power that will cause receiver loss of lock. Third, a limited number of UWB waveforms and discrete power levels were used in the previous testing reports. This limitation makes the previous testing results outdated as the UWB community continues to explore new waveforms and new modulations schemes. Finally, previous testing results are limited to a relatively small set of UWB sources. One of the most important features of UWB techniques is the potential for a large number of UWB devices to co-exist and operate in a relatively small area without interfering with each other.

What are the characteristics of a large number of aggregated UWB interference? What is the impact of the aggregated UWB devices on the performance of GPS receivers? Evidently, it is not feasible to obtain answers for these questions using experiment-based testing as was done in previous studies.

This paper presents a different approach to evaluate the impact of UWB interference on GPS receivers. Software GPS receivers are used in this study to provide greater flexibility in identifying the interference effect at various stages of the receiver design. A framework was established for developing algorithms to evaluate UWB and GPS interference under a wide variety of hardware and software conditions. This framework provides great flexibility in the design of testing schemes while allowing users to focus on the effects of specific receiver functions. Both real UWB signals and simulated UWB signals can be used in the study. The real UWB signals can be used to validate the simulation procedure. Simulated UWB signals can be used to allow immediate incorporation of new UWB waveforms and modulations in the evaluations. Using simulation also made it possible to create a large number of aggregated UWB signals and testing its impact on GPS receivers. In this study, a prototype simulated version of a radio frequency (RF) front end for a software GPS receiver developed at Air Force Research Laboratory (AFRL) Sensors Directorate, WPAFB was created. This simulated RF front end processes input UWB signals (simulated or real) and generates a digitized output. This output can then be mixed with various GPS signals (simulated or real) to undergo acquisition, tracking, and post-processing procedures. By varying the input UWB parameters, one may study the output at different stages of the GPS receiver to assess the UWB interference impact on the GPS signals at those stages.

In the remainder of this paper, a review of the current state of UWB waveform designs and modulation techniques will be given, followed by a description of the general framework established for simulating UWB interference on GPS software receiver functionality. Detailed software algorithm design for

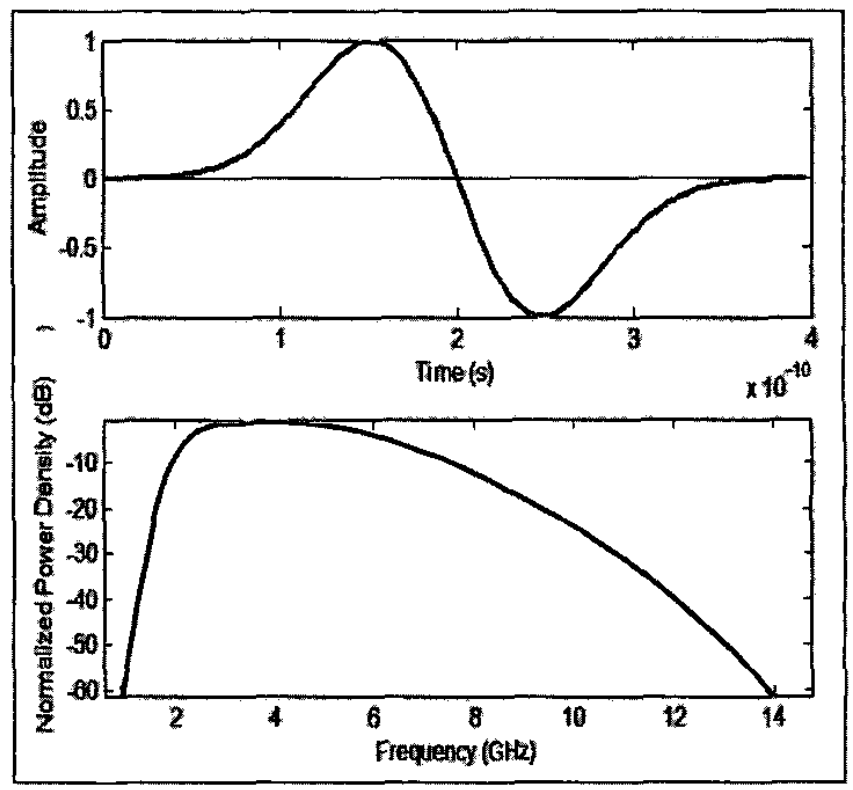

Fig. 2. Time and frequency characteristics of a Gaussian monopulse as a short impulse signal

the simulation will then be presented. The preliminary test results and future works will also be discussed.

\section{CURRENT STATE OF UWB WAVEFORM DESIGN AND MODULATION SCHEMES}

A UWB signal is one whose bandwidth exceeds $0.5 \mathrm{GHz}$ or whose $-10 \mathrm{~dB}$ bandwidth exceeds $20 \%$ of its center frequency, whichever is smaller. To protect certain restricted bands such as GPS signals from UWB interference, FCC has set maximum Effective Isotropic Radiated Power Density (EIRPD) for UWB signals at different frequency bands. Figure 1 shows this prescribed spectral mask set by the FCC. 
The fundamental building blocks of a UWB signal are impulses. The original impulse radio uses short impulses with durations that are no more than $1 \mathrm{~ns}$ [8]. Figure 2 shows the well-studied Gaussian monopulse and its normalized power spectral density. Recently, more research has been focused on synthesizing means that can generate more complex waveforms that can more effectively utilize the allotted spectrum, reduce power consumption, achieve more reliable communications, and reduce complexity in receiver hardware design [2]. The IEEE P802.15.3 High Rate (HR) Task Group (TG3) for Wireless Personal Area Networks (WPANs) is chartered to draft and publish a new standard for high-rate $(20 \mathrm{Mbit} / \mathrm{s}$ or greater) WPANs using UWB signals (http://www.ieee802.org/15/pub/TG3.html). Currently, there are two competing proposals, led by Texas Instruments and XtremeSpectrum, Inc., respectively, seeking the standardization of UWB for future WPAN devices.

The proposal led by Texas Instruments uses the Multi-Band Orthogonal Frequency Division Multi-access (OFDM) technique [9]. This proposal divides the 3.1 to $10.6 \mathrm{GHz}$ into multiple bands, each with $528 \mathrm{MHz}$ bandwidth to enable multiple modes of device operation. Information is transmitted using OFDM modulation on each band. The proposed data rate ranges from 55 to $480 \mathrm{Mbps}$ with symbol length at 312.5 ns. The proposal led by XtremeSpectrum Inc. [10] uses joint time-frequency wavelet family pulses to transmit and receive signals over two distinct bands (3.1-5.15 GHz and 5.825 to $10.6 \mathrm{GHz}$ ), with a proposed data rate from 25 to $450 \mathrm{Mbps}$. $\mathrm{M}$-ary biothogonal keying is proposed as the modulation scheme.

Since the UWB signals are still undergoing continuous debate, experimental testing of their interference with GPS receivers is not feasible. Testing of aggregated future UWB device impact on GPS receivers is even harder to realize. The software approach described below provides a feasible alternative for the interference evaluation.

\section{FRAMEWORK FOR UWB-GPS INTERFERENCE STUDIES}

As discussed previously, UWB technology is undergoing continuous change and evolution. To be able to study the UWB interference impact on GPS receiver performance in a timely manner, it is necessary to establish a general simulation framework that can provide systematic means that can be applied to a wide variety of signal and system designs. Figure 3 shows the basic framework developed for this purpose.

As shown in the figure, this basic framework consists of three main functional blocks: input, processing, and analysis. Each component in the framework can have a variety of implementations. The components interact with each other via defined interfaces. One may replace a particular component with a different internal design and implementation without affecting the set up of the rest of system. Doing so allows one to experiment with any type of data sources, signal forms, processing architecture, and analysis methods with a minimum amount of software redesign and rebuild. For example, the

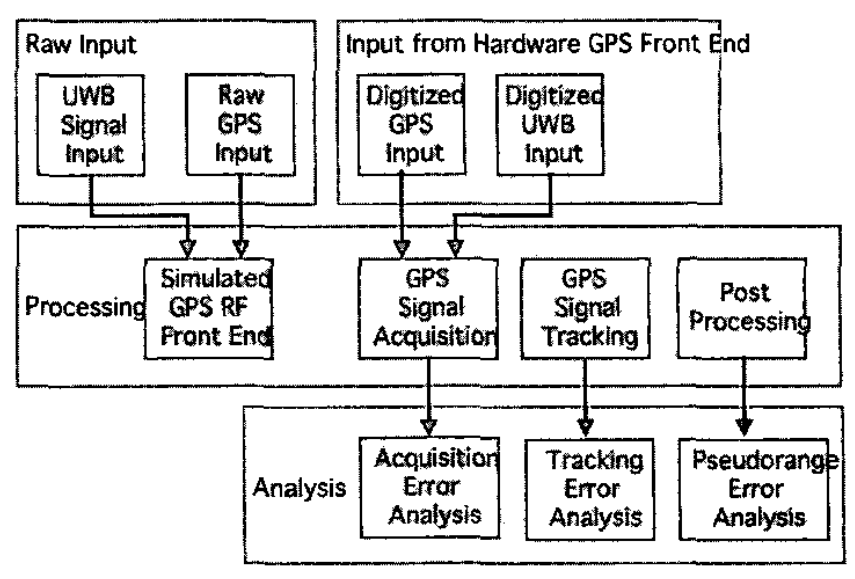

Fig. 3. General framework for UWB-GPS
interference studies

UWB signal input can be simulated waveforms and simulated modulation schemes. It can also be real samples of UWB signals generated by a UWB transmitter. Furthermore, the samples could be taken via conducted path or radiated path. The digitized GPS signal can be simple simulation data, or data generated by a GPS signal simulator, or data taken by a specific GPS RF front end. These different forms of input data give us the flexibility and capability to analyze system response under a wide range of conditions.

In software GPS receivers, some of the functions, such as acquisition and tracking, which are traditionally done using hardware, are also implemented with software. The only hardware is the radio frequency (RF) front end. Figure 4 depicts the block diagram of a typical GPS RF front end consisting of antenna, preamplifier/RF filter, down-converter, intermediate frequency (IF) filter, and analog to digital converter (ADC) $[11,12]$. An alternative design involves direct sampling of RF signals without down-conversion [13, 14]. In this project, a simulated RF front end, resembling the design used for a software GPS receiver at AFRL/WPAFB, is created. With the flexibility built into the framework, one may easily replace the simulated RF front end with a new design when needed.

The UWB output from the GPS RF front end can then be mixed with digitized GPS signals and fed to the acquisition stage. There is a number of acquisition algorithms developed over the years [15]. We will be using a software acquisition algorithm developed at AFRL/WPAFB.

The output of the acquisition stage will be analyzed to study the UWB signal's threshold EIPRD at which acquisition fails. This study can be done for a range of GPS signal power levels and various GPS signal conditions. It can also be done for any combination of UWB waveforms and modulation methods.

The AFRL at WPAFB has also developed tracking algorithms for both nominal and weak signals, and post processing algorithms. These algorithms can be used to test the output at each of these processing blocks to determine the UWB impact. Evaluation of tracking algorithms is an on-going project. 


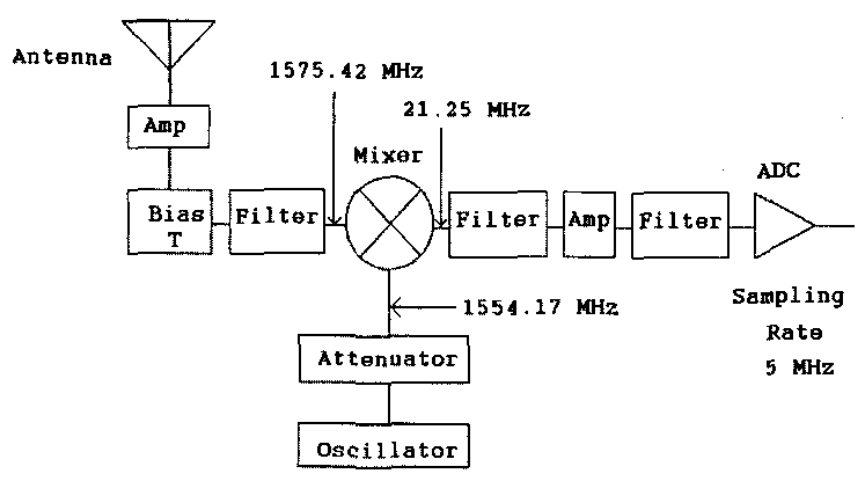

Fig. 4. A GPS RF front end

Acquisition, tracking, and post processing algorithms developed at other research and industrial organizations could also be used for testing using this framework. Doing so allows one to evaluate the receiver performance for a wide variety of applications such as the impact of UWB on weak GPS signals, signals under high dynamic conditions, and different approaches in algorithms. New types of UWB sources can be tested and their impact can be evaluated before they are approved or deployed. Furthermore, one may simulate and test the effect of a theoretical UWB waveform before it is realized in hardware.

\section{SIMULATION RESULTS}

A Gaussian monopulse is used to test the GPS front end simulation results. Figure 7 shows the input UWB impulse $\mathrm{x}(\mathrm{t})$ and the output at each stage of the simulated front end.

In Figure 5, the horizontal axis is time in unit of $\mu \mathrm{s}$. A single pulse with amplitude of $1 \mathrm{~V}$ and pulse width of 250 picoseconds is used in the simulation. As can be seen from the figure, the antenna output consists of a delayed ringing response of the input pulse, as expected. The down conversion process took some of the higher frequency ringing effect out of the antenna output. The IF filter further filtered out the extreme frequency components and also introduced a delay in the response. For an input with amplitude of $1 \mathrm{~V}$, the final sampled output has a peak around $0.2 \mathrm{mV}$, a 5000 fold reduction in signal strength.

What happens when a sequence of UWB signals are received? How do the impulses interact with each other? Considering that the system being simulated is a linear-time invariant (LTI) system, the response of the system to a sequence of pulses should be the superposition of each individual pulse response, taking into consideration the time delays between each of the pulses. To verify that such an assumption is valid, we generated a sequence of UWB pulses and obtained the simulation output of the sequence at the front end. The result is then compared to the superposition of each individual pulse output with the same time delay. The results are identical. Figure 6 shows the result of a sequence of UWB impulses. The parameters of each individual pulses are the same as those used to generate Figure 5. The variations in the input amplitude in the figure are due to sampling. The

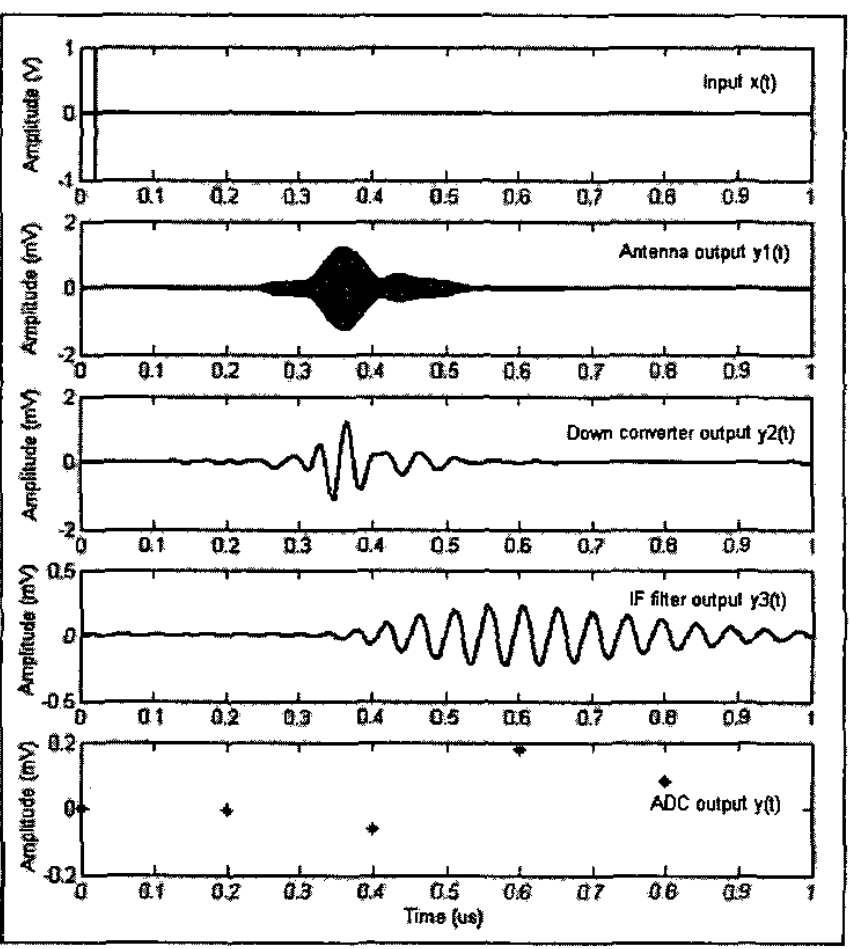

Fig. 5. A UWB impulse and its output at each stage of the simulated GPS front end

sequence uses an absolute random dithering modulation scheme. The PRF is $1 \mathrm{MHz}$, and the dithering can be up to $50 \%$ of $1 /$ PRF.

One of the difficulties associated with the simulation is the size of the input, intermediate, and output signal vectors. Since the simulation output will be combined with the GPS input to undergo the acquisition procedure, at least $1 \mathrm{~ms}$ of continuous data is needed for the processing. With a sampling rate of 20 $\mathrm{GHz}, 1 \mathrm{~ms}$ input data consists of 20 million samples. This size is beyond what Matlab can handle. Considering the case of weak GPS signal acquisition, several hundred milliseconds of data may be used for acquisition alone. It is necessary to come up with methods that can break the front end simulation into small segments of data, and then combine them at the output to make the processing feasible.

Table 1. Software GPS Receiver Acquisition Success Rate

\begin{tabular}{|c|c|c|c|c|c|c|c|c|c|c|c|}
\hline \multirow{2}{*}{ Amp. } & \multicolumn{10}{|c|}{ Pulse Repetition Rate (MHz) } \\
\cline { 2 - 12 } & 1 & 2 & 5 & 10 & 25 & 55 & 110 & 160 & 200 & 320 & 420 \\
\hline $0.1 \mathrm{~V}$ & $98 \%$ & $98 \%$ & $98 \%$ & $98 \%$ & $98 \%$ & $97 \%$ & $98 \%$ & $97 \%$ & $97 \%$ & $93 \%$ & $94 \%$ \\
\hline $0.2 \mathrm{~V}$ & $99 \%$ & $98 \%$ & $98 \%$ & $97 \%$ & $97 \%$ & $96 \%$ & $92 \%$ & $89 \%$ & $85 \%$ & $82 \%$ & $69 \%$ \\
\hline
\end{tabular}




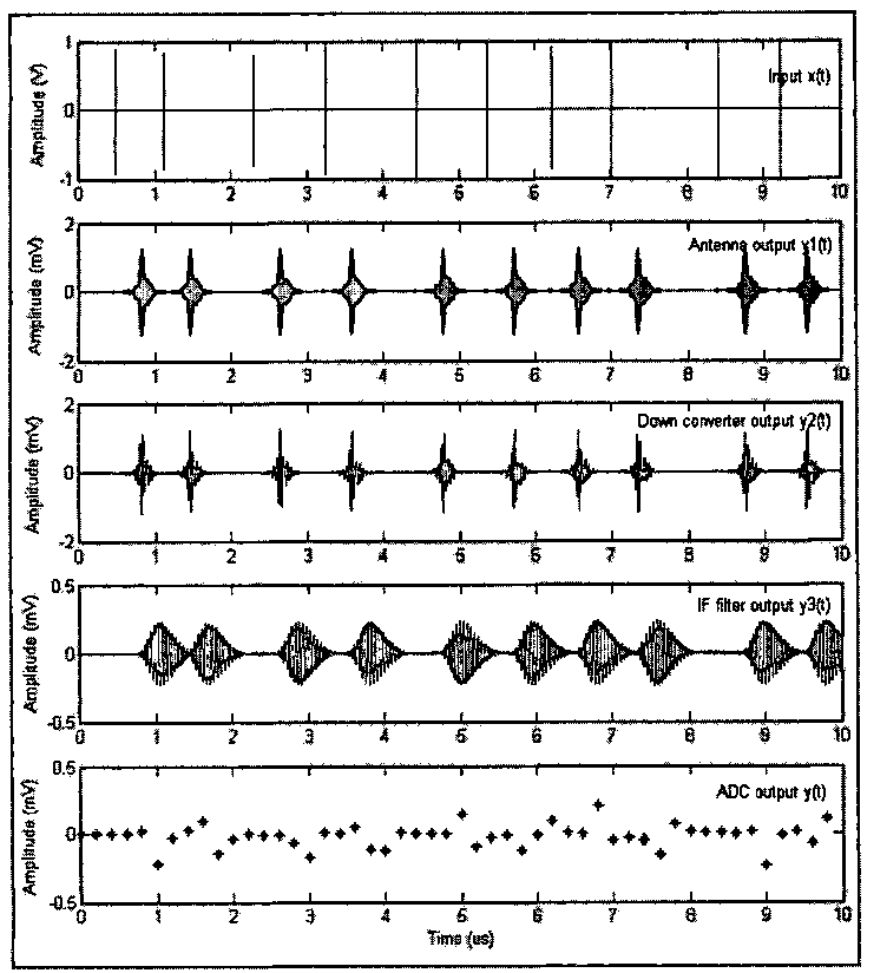

Fig. 6. A sequence of UWB impulse and their outputs at each stage of the simulated GPS front end

A signal superposition method is used to solve this problem. First, we need to know how long it takes for the output of an impulse at the GPS front end to become negligible. We found that this time is determined by the width of the narrow bandpass filter in the front end design. For a bandwidth $\mathrm{BW}=$ $2 \mathrm{MHz}$, this time constant is no more than $10 / \mathrm{BW}=5 \mu \mathrm{s}$. To be conservative, $10 \mu \mathrm{s}$ is used in our calculation. An algorithm is developed to process long sequences of UWB impulses:

1. Divide the input UWB signals into blocks. Each block has a length of $20 / \mathrm{BW}=10 \mu \mathrm{s}$.

2. Take the first half of a block, pad the second half with zeros. Process such a block to generate an output sequence. Divide the output sequence into two equal parts: part1 and part2. The output corresponding to the first part (denoted as out1) is the sum of the current block part 1 and the previous blocks part 2 .

3. Write out1 to data file.

\section{Repeat the above process.}

We tested the above algorithm using two $10 \mu$ s block length and found that the output is the same as the result of processing one $20 \mu$ s block. Using this approach, we were able to process very long sequences of UWB data and store the data for the acquisition stage.

A preliminary test using the simulation model was conducted to examine the impact of a Gaussian monopulse type UWB signal on the software receiver acquisition performance. The UWB power spectral density is a function of both the pulse amplitude and the pulse repetition rate. Table 1 summarizes the success rate of the software GPS receiver acquisition function when simulated GPS and simulated UWB Gaussian monopulses are mixed together as input.

A total of eight simulated satellite signals are included in the GPS inputs. The signal to noise ratio of all eight satellites is $-15 \mathrm{~dB}$ (referenced to the $2 \mathrm{MHz}$ GPS CA code bandwidth). The Doppler frequency, carrier phase, and initial CA code phases of the GPS signals are randomly generated within their respective allowable ranges. The average successful rate for the software GPS receiver acquisition is $99 \%$ when UWB signals are not present in the input.

UWB pulses with pulse width of 250ps are generated for the simulation. Two different values of the UWB pulse amplitudes are tested: $100 \mathrm{mV}$ and $200 \mathrm{mV}$. For each amplitude value, pulse repetition rates ranging from $1 \mathrm{MHz}$ to $420 \mathrm{MHz}$ are used to generate a sequence of UWB pulses. Random dithering within $50 \%$ of the repetition interval is assumed when generating the pulse sequence. Signals from a total of 100 UWB sources located 6 feet away from the GPS receiver are added to the simulated GPS RF front end. Each data point in Table 1 is the average result of 100 simuiation runs. The success rate as shown in the table indicates that for a given pulse amplitude, as the repetition rate increases the acquisition rate declines. The decline is accelerated when the pulse repetition rate approaches the high end of the projected data rate for UWB applications.

\section{CONCLUSIONS AND FUTURE PLANS}

This paper presented a simulation model for the GPS RF front end. This simulation model consists of a GPS antenna, RF to IF conversion, CA code bandpass filter, and analog to digital converter. This simulation model incorporated a variety of elements such as the use of experimental data (GPS antenna frequency response), mathematical modeling (down conversion), and signal processing tool boxes (bandpass filter). Several other necessary components were also created in conjunction with the RF front end model. These include the generation of UWB pulses and the algorithm used to handle long sequences of input data.

The simulation model established through this study will provide a framework for studying the impact of any combination of future UWB signals on GPS receivers at various stages. It has the advantage of having the flexibility to work with a variety of GPS receiver architectures and allows a closer look into the fundamental aspects of interference and performance degradation. It can be a valuable tool for the GPS and UWB community to study a signal of interest before deploying it into the mass market. 
There are several follow up projects:

1. GPS RF front end simulation model validation using real UWB signals.

2. UWB source simulation. As new UWB sources are being proposed, approved, and implemented, we can simulate these signals and use them in the interference evaluations.

3. More acquisition stage impact analysis. The preliminary results only examined the interference of the Gaussian monopulse using random dithering as the modulation scheme. More realistic signals and modulation schemes should be used in future research.

4. Interference study at the tracking and post processing stages.

\section{ACKNOWLEDGEMENTS}

This project was supported by an AFOSR summer faculty fellowship award and AFOSR/AFRL under contract \#F49620-03-1-0225. The authors thank the following organizations and individuals for their help and support for this project:

- Kenneth Walker of the Sensors Directorate, AFRL/SNRW, for providing access to Multispectral Solutions TFP 1001 UWB signal generator.

- Steven Hary of the Sensors Directorate, AFRL/SNRP, for editing the manuscript and providing valuable suggestions and comments to this work, and

- Boyd Holsapple, Pete Howe, and Liyeh Liou of the Sensors Directorate, AFRL/SNRP, for their help and support during this project.

\section{REFERENCES}

[1] Federal Communications Commission, First Report and Order ET Docket 98-153, Revision of Part 15 of the Commission's Rules Regarding Ultra-Wideband Transmission Systems, adopted February 14, 2002.

[2] Leeper, D.G., June 2003 , Ultrawideband - The Next Step in Short-Range Wireless, IEEE 2003 IMS/RFIC Symposium, pp. 357-360, June, 2003.

[3] Aiello, G.R., June 2003,

Challenges for Ultra-wideband (UWB) CMOS Integration, IEEE 2003 IMS/RFIC Symposium, pp. 361-364, June, 2003.
[4] Anderson, D.S., Drocella, E.F., Jones, S.K. and Settle, M.A., February 2001,

Assessment of compatibility between Ultrawideband systems and Global Positioning System (GPS) Receivers, NTIA Special Publication 01-45, Washington DC, Feburary, 2001, Internet URL: http://www.ntia.doc.gov/osmhome/reports/ UwbGps/NTIASP_01_45.pdf.

[5] Titus, B.M., Dafesh, P.A., Wong, R., Maine, K.P. and Stansesll, T.A., Jr., September 11-14, 2002, Assessing Ultra Wide Band (UWB) interference to GPS receivers, Proceedings of 2002 Institute of Navigation GPS Conference, Portland, Oregan, September 11-14, 2002, pp. 1251-1259.

[6] Eriandson, R.J. and Van Dierendonck, A.J., September 11-14, 2001, GPS receiver susceptibility to ultra-wideband RFI: test results and RFI link analysis,

Proceedings of 2001 Institute of Navigation GPS Conference, Salt Lake City, UT, September 11-14, 2001, pp. 14-25.

[7] Luo, M., Akos, D., Koenig, M., Opshaug, G., Pullen, S. and Enge P., September 11-14, 2001,

Testing and research on interference to GPS from UWB transmitters, Proceedings of 2001 Institute of Navigation GPS Conference, Salt Lake City, UT, September 11-14, 2001, pp. 1-13.

[8] Win, M.Z. and Scholtz, R.A., January 1998, Impulse radio: HOW IT WORKS, IEEE Communications Letters, Vol. 2, No. 1, pp. 10-12, January 1998.

[9] Aiello, G.R., et al., http://www.multibandofdm.org/papers/15-04-0010-02-003a-multiband-ofdm-jan04-presentation.pdf.

[10] Melbprn, M., et al., $\mathrm{ftp}: / / \mathrm{ftp} .802$ wirelessworld.com/15/03/15-03-0334-03-003axtremespectrum-cfp-presentation.pdf.

[11] Van Dierendonck, A.J., 1996, Chapter 8. GPS Receivers, in Global Positioning System: Theory and Applications, Volume 1, edited by B.W. Parkinson and J.J. Spilker, Jr., published by AIAA, 1996.

[12] Tsui, J.B., 2000, Fundamentals of Global Positioning System Receivers, A Software Approach, John Wiley \& Sons, 1st edition, 2000.

[13] Akos, D.M. and Tsui, J.B.Y., 1996, Design and implementation of a direct digitization GPS receiver front end,

IEEE Tran. On Microwave Theory and Techniques, Vol. 44, No. 12, pp. 2334-2339, 1996.

[14] Akos, D. and J. Thor, April 2002, A direct RF sampling multifrequency GPS receiver, IEEE Position Location and Navigation Symposium, Palm Springs, CA, April, 2002.

[15] Lin, D.M. and Tsui, J.B.Y., 2000,

Comparison of Acquisition Methods for Software GPS Receivers, Proceedings of Institute of Navigations GPS Conferences, pp. 2385-2390, Salt Lake City, Utah, 2000. 